cristiano alessandri Grant/research support from: Pfizer, Guido Valesini: None declared, fabrizio conti Speakers bureau: BMS, Lilly, Abbvie, Pfizer, Sanofi DOI: 10.1136/annrheumdis-2020-eular.4245

\begin{tabular}{|l|l}
\hline FRI0160 & THE CORRELATION BETWEEN PREGNANCY, \\
& DISEASE ACTIVITY AND ADVERSE PREGNANCY \\
& OUTCOMES IN PATIENTS WITH SYSTEMIC LUPUS \\
& ERYTHEMATOSUS
\end{tabular}

C. Cetin $^{1}$, T. Saraç-Sivrikoz ${ }^{2}$, M. Ateş-Tıkız ${ }^{2}$, E. S. Torun ${ }^{1}$, S. Zaralı ${ }^{1}$, Y. Yalçınkaya ${ }^{1}$, A. Gul ${ }^{1}$, M. Inanc ${ }^{1}$, M. L. Ocal' ${ }^{1}$, I. Kalelioğlü ${ }^{2}$, B. Artim-Esen ${ }^{1}$. ${ }^{1}$ Istanbul Faculty of Medicine, Rheumatology, Istanbul, Turkey; ${ }^{2}$ Istanbul Faculty of Medicine, Gynecology and Obstetrics, Istanbul, Turkey

Background: Patients with systemic lupus erythematosus (SLE) can present with acute disease flares/exacerbations during pregnancy and postpartum period. ${ }^{1}$ These flares can cause adverse pregnancy outcomes (APO).

Objectives: In this study, our pregnant SLE cohort, which was under medical surveillance of both our Rheumatology and Gynecology and Obstetrics departments was analyzed. We intended to determine the effects of pregnancy on disease activity and the correlation between disease flares and adverse pregnancy outcomes.

Methods: 168 pregnancy data involving 136 patients with SLE meeting the ACR criteria were examined. Cumulative clinical, laboratory and serological parameters were described and disease activity and flares were calculated using SLEDAI-2K disease activity index during preconceptional six month period, during all trimesters of pregnancy, and during postpartum six month period. Patients with low lupus disease activity scores (LLDAS) during each of these periods were identified. Fetal/neonatal death, premature birth due to preeclampsia, eclampsia or HELLP syndrome, neonates small for gestational age were determined as adverse pregnancy outcomes. Relationship of APO with disease activity was studied and patients with APO were compared to patients without APO.

Results: Mean SLEDAI-2K scores was $1.3 \pm 2.2(0-16)$ during preconceptional six month period, $1.3 \pm 2.6(0-16)$ during conception period, $1.7 \pm 3.2(0-22)$ during first trimester, $1.4 \pm 2.7(0-16)$ during second trimester, $1.5 \pm 3.3(0-20)$ during third trimester and $3.5 \pm 5.4(0-26)$ during postpartum six month period. Mean postpartum six month period SLEDAI-2K score was higher compared to the mean pregnancy SLEDAI-2K score $(\mathrm{p}<0.05)$. LLDAS was sustained in $79 \%$ of all pregnancies. $19 \%$ of pregnancies resulted in flares. $42 \%$ of these flares were severe and $58 \%$ were mild or moderate. $49 \%$ of severe flares occurred during the postpartum six month period and this percentage was significantly higher compared to each trimester $(\mathrm{p}<0.05)$. Most of the flares during pregnancy and postpartum period had mucocutaneous (37\%), renal(35\%) and hematological(25\%) involvement. APO was observed in $34 \%$ of pregnancies $(n=57)$. APO (+) group was characterized by significantly longer disease duration and higher disease activity in all periods compared to APO (-) group $(142 \pm 70$ vs $170 \pm 88$ months, $p<0.05)$. In APO (-) group, the proportion of patients with severe disease activity during all pregnancy periods and postpartum period was significantly low (\%18 vs $35, p<0.05)$, while the proportion of patients with sustained LLDAS was much higher (\%88 vs 70$)$.

Conclusion: Postpartum six-month period appears to have the highest risk for disease flares during SLE pregnancies. Disease activity during pregnancy increases the risk of APO. Patients with sustained LLDAS have significantly lower APO rates. In order to achieve a positive pregnancy outcome and lower maternal morbidity, regular follow up of patients during pregnancy and postpartum period by Rheumatology and Gynecology and Obstetrics Departments is necessary. References:

[1] Eudy AM, et al. Ann Rheum Dis 2018;0:1-6. doi:10.1136/annrheumdis2017-212535

Disclosure of Interests: None declared

DOI: 10.1136/annrheumdis-2020-eular.2482

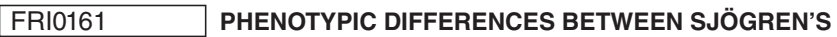 SYNDROME PATIENTS WITH LOW AND HIGH-GRADE INFLAMMATION BASED ON SALIVARY GLAND FOCUS SCORE}

L. Chatzis ${ }^{1}$, V. Pezoulas ${ }^{2}$, F. Ferro ${ }^{3}$, V. Donati ${ }^{3}$, A. Venetsanopoulou ${ }^{1}$, E. Zampeli ${ }^{4}$, M. Mavromati ${ }^{4}$, P. Voulgari ${ }^{5}$, C. Mavragani ${ }^{1}$, D. Fotiadis ${ }^{2}$, F. Skopouli ${ }^{4}$, S. De Vita ${ }^{6}$, G. Vassilis ${ }^{7}$, C. Baldini ${ }^{3}$, H. M. Moutsopoulos ${ }^{4}$, A. Goules ${ }^{1}$, A. Tzioufas ${ }^{1} .{ }^{1}$ Pathophysiology Department, Athens School of Medicine, National and Kapodistrian University of Athens, Athens, Greece; ${ }^{2}$ Unit of Medical Technology and Intelligent Information Systems, University of loannina, loannina, Greece; ${ }^{3}$ Rheumatology Unit, Department of Clinical and Experimental Medicine, University of Pisa, Pisa, Italy; ${ }^{4}$ Department of Nutrition and Clinical Dietetics, Harokopio University of Athens, Athens, Greece;
${ }^{5}$ Rheumatology Clinic, Department of Internal Medicine, Medical School, University of loannina, Ioannina, Greece; ${ }^{6}$ Rheumatology Clinic, Department of Medical area, University of Udine, Udine, Italy; ${ }^{7}$ Laboratory of HistologyEmbryology Molecular Carcinogenesis Group Medical School National and Kapodistrian University of Athens, Athens, Greece

Background: Sjögren's syndrome (SS) is characterized by the presence of lymphocytic infiltration around the ductal epithelium of the salivary and lachrymal glands. The periepithelial inflammatory lesions and the enclosed B cell component are responsible for the glandular and extraglandular manifestations of the disease. Previous studies have shown that the severity of inflammation observed within the salivary glands is correlated with the occurrence of extraglandular manifestations. However, in these studies either the number of patients is small or the SS criteria are not well defined. To explore the association between the degree of inflammation within the salivary glands and the phenotype of the disease, large and well characterized cohorts of SS patients is required.

Objectives: To compare the phenotypic features of SS patients with low and high degree of inflammation within the minor salivary glands as reflected by the focus score (FS).

Methods: From a total cohort of 1723 consecutive SS patients who fulfill the 2016 EULAR/ACR criteria and are followed up in 4 clinical centers ([Universities of Pisa, Athens, Harokopio and Ioannina, (PAHI)], those who had performed a lip biopsy and the focused score was available, were classified into low grade $(F S<3)$ or high grade $(F S \geq 3$ ). Glandular (dry mouth, dry eyes, parotid gland enlargement) and extra-glandular manifestations (Raynaud's phenomenon, arthralgias/ myalgias, arthritis, palpable purpura, liver involvement, kidney involvement, lung involvement, neurologic involvement, long standing lymphadenopathy and lymphoma) as well as serologic features (ANA, RF, anti-Ro/SSA, anti-La/SSB) were compared between the 2 groups. Statistical analysis for categorical variables was performed by Fisher exact or chi-square tests and for continuous variables with t test or Mann-Whitney accordingly.

Results: Eight hundred and eight minor salivary gland biopsies were available and evaluated based on focus score at the initial evaluation of SS patients, of whom 753 had low grade $(F S<3)$ and 153 high grade $(\geq 3)$ inflammation. The median disease duration after SS diagnosis was not statistically significant different for the 2 groups (median: 4 years, range: 0-36 years). SS patients with high grade inflammation displayed higher prevalence of salivary gland enlargement (SGE) $(40 \%$ vs $25 \%, p=0,0002)$, long standing lymphadenopathy ( $22 \%$ vs $14 \%$ $p=0,02)$, ANA ( $97 \%$ vs $88 \%, p=0,0001)$, anti-La/SSB ( $52 \%$ vs $32 \%, p<0,0001)$ RF $(61,5 \%$ vs $48 \%, p=0,003)$, peripheral neuropathy (PN) $(5,3 \%$ vs $1,5, p=0,01)$ and of lymphoma ( $26 \%$ vs $8 \%, p<0,0001, O R=4,142,95 \% \mathrm{Cl}=2,65$ to 6,47$) \mathrm{com}$ pared to those with low grade inflammation.

Conclusion: SS patients with $\mathrm{FS} \geq 3$ at the initial evaluation, display higher prevalence of lymphoma as well as higher B cell hyperactivity and certain clinical manifestations (SGE, PNS, lymphadenopathy) that constitute risk factors for lymphoma development.

Disclosure of Interests: Loukas Chatzis: None declared, Vasileios Pezou las: None declared, Francesco Ferro: None declared, Valentina Donati: None declared, Aliki Venetsanopoulou: None declared, Evangelia Zampeli: None declared, Maria Mavromati: None declared, Paraskevi Voulgari: None declared Clio Mavragani: None declared, Dimitris Fotiadis: None declared, Fotini Skopouli: None declared, Salvatore De Vita Consultant of: Roche, Human Genome Science, Glaxo Smith Kline and Novartis, Gorgoulis Vassilis: None declared, Chiara Baldini: None declared, Haralampos M. Moutsopoulos: None declared, Andreas Goules: None declared, Athanasios Tzioufas: None declared

DOI: 10.1136/annrheumdis-2020-eular.4335

\section{FRI0162 COMPARISON OF MUSCULOSKELETAL INVOLVEMENT OF WRIST AND HAND BY MRI IN TWO SYSTEMIC LUPUS ERYTHEMATOSUS GROUPS: HAND ARTHRITIS AND HAND ARTHRALGIA}

$\underline{\text { P. Corzo }}^{1}$, I. Garcia-Duitama ${ }^{2}$, A. Agustí Claramunt ${ }^{2}$, T. C. Salman Monte ${ }^{3}$, J. Monfort ${ }^{3} .{ }^{1}$ Hospital Plató, Rheumatology, Barcelona, Spain; ${ }^{2}$ Hospital del Mar, Radiology, Barcelona, Spain; ${ }^{3}$ Hospital del Mar, Rheumatology, Barcelona, Spain

Background: Articular involvement can reach up to $95 \%$ within the multisystemic manifestations of SLE. Originally, a non-erosive pattern of articular inflammation was described, but the emergence of more sensitive imaging techniques, such as Magnetic Resonance Imaging (MRI), show synovitis (S), erosions (E), bone marrow edema (BME) and tenosynovitis (TS) in patients with systemic lupus erythematosus (SLE). Nowadays, a specific validated pattern of articular involvement associated with this disease does not yet exist, although it has begun to be studied $(1,2)$. 